\title{
Iterative Amplitude/Phase Multiple-Symbol Differential Sphere Detection for DAPSK Modulated Transmissions
}

\author{
Li Wang* Member, IEEE, K. V. S. Hari ${ }^{\diamond}$ Senior Member, IEEE, and Lajos Hanzo*, Fellow, IEEE
}

\begin{abstract}
Differentially encoded and non-coherently detected transceivers exhibit a low complexity, since they dispense with complex channel estimation. Albeit this is achieved at the cost of requiring an increased transmit power, they are particularly beneficial, for example in cooperative communication scenarios, where the employment of channel estimation for all the mobile-to-mobile links may become unrealistic. In pursuit of high bandwidth efficiency, differential amplitude and phase shift keying (DAPSK) was devised using constellations of multiple concentric rings. In order to increase resilience against the typical high-Doppler-induced performance degradation of DAPSK and/or enhance the maximum achievable error-free transmission rate for DAPSK modulated systems, multiple-symbol differential detection (MSDD) may be invoked. However, the complexity of the maximuma-posteriori (MAP) MSDD increases exponentially with the detection window size and hence may become excessive upon increasing the window size, especially in the context of iterative detection aided channel coded system. In order to circumvent this excessive complexity, we conceive a decomposed two-stage iterative amplitude and phase (A/P) detection framework, where the challenge of having a non-constantmodulus constellation is tackled with the aid of a specifically designed information exchange between the independent $\mathrm{A} / \mathrm{P}$ detection stages, thus allowing the incorporation of reduced-complexity sphere detection (SD). Consequently, a near-MAP-MSDD performance can be achieved at a significantly reduced complexity, which may be five orders of magnitude lower than that imposed by the traditional MAP-MSDD in the 16-DAPSK scenario considered.
\end{abstract}

\section{INTRODUCTION}

D IFFERENTIAL phase shift keying (DPSK) relying on lowcomplexity non-coherent detection constitutes an attractive solution for wireless communications, especially in scenarios, such as for example, cooperative communications, since it is robust against the phase ambiguities induced by rapid fading, while dispensing with complex timing recovery and channel estimation for mobileto-mobile links. For the sake of transmitting an increased number of bits/symbol, differential amplitude and phase shift keying (DAPSK) [1-3] was proposed, which expands the single-ring constellation of traditional DPSK to multiple rings. Essentially, the information is encoded both in the amplitude- and phase-differences between successively transmitted symbols.

In order to enhance the maximum achievable error-free transmission rate for a given DAPSK modulation as well as to eliminate the typical emergence of an error-floor at high Doppler-frequencies, the powerful multiple-symbol differential detector (MSDD) has been applied to uncoded DAPSK-modulated systems in [4], which relies on the joint detection of multiple consecutively received symbols. However, when employed in an iterative detection aided channel coded DAPSK-aided system, the maximum-a-posteriori (MAP) softdecision MSDD [5,6] employing even a moderate observation window size $N$ may still exhibit an excessive complexity, since it has to generate soft information based on the brute-force search for every transmitted bit. As a potential complexity reduction technique, the well-known tree-search-based sphere detection (SD) mechansim has been proposed for MSDD of a conventional DPSK modulated system [7]. This solution was termed as multiple-symbol differential

*University of Southampton, UK (e-mail: $\{1 \mathrm{w} 5, \mathrm{lh}\} @$ @ecs.soton.ac.uk).

${ }^{\diamond}$ Indian Institute of Science, India (e-mail: hari@ece.iisc.ernet.in).

The research leading to these results has received funding from the European Union's Seventh Framework Programme ([FP7/2007-2013]) under grant agreement no [214625]. The finacial support of the RC UK under the auspices of the UK-India Advanced Technology Centre of Wireless Communications and of the China-UK Science Bridge in $4 \mathrm{G}$ wireless communications, as well as that of the EU's Optimix project is also gratefully acknowledged. sphere detection (MSDSD). Unfortunately, the non-constant-modulus constellation DAPSK precludes the direct application of the MSDSD scheme of [7]. Thus, until now the conception of an efficient MSDD for DAPSK-aided systems remained an open problem.

Against this background, firstly, we close this open problem by proposing an iterative $A / P$ detection framework for $M S D D$-aided DAPSK systems; Secondly, the iterative information exchange between the above-mentioned $A / P$ detection stages is specifically tailored for mitigating any potential performance penalty imposed by the separate A/P detection; Thirdly, we incorporate the SD mechanism in this new MSDD for the sake of further complexity reduction. Our simulation results demonstrate a near-MAP-MSDD performance can be achieved at a significantly reduced complexity, which may be five orders of magnitude lower than that imposed by the traditional MAP$M S D D$ in the 16-DAPSK scenario considered.

\section{System Architecture \& Channel Model}

The simplified overall system model of bit-interleaved coded differential modulation is depicted in Fig. 1, where 16-DAPSK is assumed to be employed without loss of generality. At the transmitter of Fig. 1, a block of $L$ information bits $u$ is first encoded by the channel encoder in order to generate the coded bits $c$, which are then interleaved by the interleaver $\pi$. The resultant permuted bits $b$ are then fed through the DAPSK modulator. The $2^{p}$-DAPSK, also known as the Star Quadrature Amplitude Modulation (Star-QAM) scheme [1], employs multiple concentric rings by combining the $2^{q}$-DASK and $2^{(p-q)}$-DPSK modulation schemes. Specifically, as illustrated in Fig. 1, the first $q$ bits, $\mathbf{b}_{\gamma}^{n}=\left[b_{\gamma, 1}^{n}, \cdots, b_{\gamma, q}^{n}\right]$, of the $n$th $p$-bit encoded APSK symbol $d[n]=\gamma[n] v[n]$ are mapped to one of the legitimate radii $\mathcal{R}=\left\{\alpha^{i_{\mathrm{A}}} \mid i_{\mathrm{A}}=0, \cdots, 2^{q}-1\right\}$ in order to generate the component ASK symbol $\gamma[n]$, for example, according to the mapping schemes of Table I. Meanwhile, the remaining $(p-q)$ bits, $\mathbf{b}_{\theta}^{n}=\left[b_{\theta, 1}^{n}, \cdots, b_{\theta, p-q}^{n}\right]$, are mapped to the component PSK symbol $v[n]=\mathrm{e}^{j \theta[n]} \in \mathcal{V}=\left\{\mathrm{e}^{j 2 \pi i_{\mathrm{P}} / 2^{(p-q)}} \mid i_{\mathrm{P}}=0, \cdots, 2^{(p-q)}-1\right\}$. Based on the above ASK and PSK modulation, differential encoding of the resultant APSK symbol $d[n]$ may be performed similarly to the conventional DPSK process in order to generate the DAPSK symbol $x[n]=a[n] s[n]$ as follows:

$$
\begin{aligned}
x[n]= & d[n] \odot x[n-1]=\gamma[n] v[n] \odot a[n-1] s[n-1], \\
= & \alpha^{\left(i_{\mathrm{A}}\{\gamma[n]\}+i_{\mathrm{A}}\{a[n-1]\}\right) \bmod 2^{q}} \\
& \cdot \exp \left[j 2 \pi\left(i_{\mathrm{P}}\{v[n]\}+i_{\mathrm{P}}\{s[n-1]\}\right) / 2^{(p-q)}\right],
\end{aligned}
$$

where $i_{\mathrm{A}}\{\cdot\}$ and $i_{\mathrm{P}}\{\cdot\}$ are the indices of the radius- and phasearguments, respectively. We note that with the aid of the modulo- $2^{q}$ operation, the transmitted component DASK symbol $a[n]$ is restricted to be taken from the same signal set as the ASK symbol $\gamma[n]$, i.e., $a[n] \in \mathcal{A}=\mathcal{R}$, as usual for DPSK, where we have $s[n] \in \mathcal{S}=\mathcal{V}$ due to the inherent periodicity of the phase. For example, the signal constellation set $\mathcal{X}$ of 16-DAPSK $(p=1, q=4)$ is constituted of two concentric rings of 8-PSK symbols.

For simplicity we consider narrow-band time-selective Rayleigh fading channels, where the fading coefficients have an autocorrelation function of $\varphi[\kappa] \triangleq \mathcal{E}\left\{h[n+\kappa] h^{*}[n]\right\}=J_{0}\left(2 \pi f_{d} \kappa\right)$, according to the widely-used Clarke model [8], with $J_{0}(\cdot)$ and $f_{d}$ denoting the zero-order Bessel function of first kind and the normalized Doppler frequency, respectively. Thus, we have the transmission model of $y[n]=h[n] x[n]+w[n]$, where the fading coefficient $h[n]$ and 


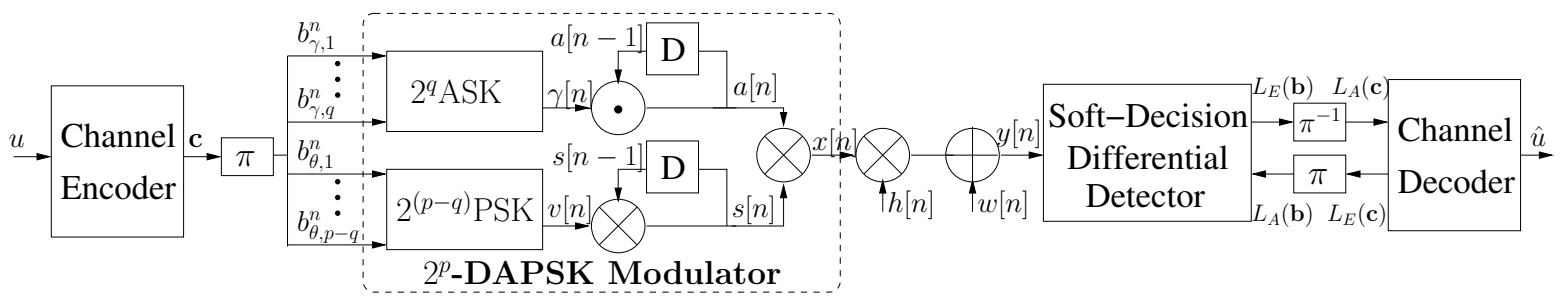

Fig. 1. Overall system model of bit-interleaved coded 16-DAPSK over Rayleigh-fading channel.

TABLE I

AMPLITUDE MAPPING FOR 16- AND 64-DAPSK

\begin{tabular}{|c|c|c|c|c|c|c|c|c|c|}
\hline \multicolumn{4}{|c|}{ 16-DAPSK $(q=1)$} & \multicolumn{6}{|c|}{ 64-DAPSK $(q=2)$} \\
\hline \multirow{4}{*}{\multicolumn{2}{|c|}{$a[n]$}} & \multicolumn{2}{|c|}{$b_{\gamma, 1}^{n}$} & \multirow{4}{*}{\multicolumn{2}{|c|}{$a[n]$}} & \multicolumn{4}{|c|}{$b_{\gamma, 1}^{n}, b_{\gamma, 2}^{n}$} \\
\hline & & \multirow{3}{*}{$\begin{array}{c}0 \\
\gamma \\
1\end{array}$} & \multirow{2}{*}{$\begin{array}{l}1 \\
n\rfloor\end{array}$} & & & 00 & 01 & 11 & 10 \\
\hline & & & & & & \multicolumn{4}{|c|}{$\gamma[n]$} \\
\hline & & & $\alpha$ & & & 1 & $\alpha$ & $\alpha^{2}$ & $\alpha^{3}$ \\
\hline \multirow{4}{*}{$a[n-1]$} & 1 & 1 & 0 & \multirow{4}{*}{$a[n-1]$} & $\overline{11}$ & $\overline{11}$ & $\overline{\alpha \alpha}$ & $\overline{\alpha^{2}}$ & $\overline{\alpha^{3}}$ \\
\hline & \multirow{3}{*}{$\alpha$} & 1 & $\alpha$ & & $\alpha$ & $\alpha$ & $\alpha^{2}$ & $\alpha^{3}$ & 1 \\
\hline & & \multirow{2}{*}{$\alpha$} & \multirow{2}{*}{1} & & $\alpha^{2}$ & $\alpha^{2}$ & $\alpha^{3}$ & 1 & $\alpha$ \\
\hline & & & & & $\alpha^{3}$ & $\alpha^{3}$ & 1 & $\alpha$ & $\alpha^{2}$ \\
\hline
\end{tabular}

the AWGN noise $w[n]$ obey a complex Gaussian distribution of $\mathcal{C N}\left(0, \sigma_{h}^{2}\right)$ and of $\mathcal{C N}\left(0,2 \sigma_{w}^{2}\right)$, respectively. Then, the received symbol $y[n]$ is processed by the turbo receiver of Fig. 1 constructed by serially concatenating the differential detector and the channel decoder, and then exchanging extrinsic information between them.

\section{Iterative AmPlitude/Phase MUltiPle-Symbol DIFFERENTIAL DETECTION}

Conventional differential detection (CDD) techniques [5] proposed for DAPSK rely on the direct calculation of the amplitude and phase differences, namely on $\lambda[n]=|y[n]| /|y[n-1]|$ and $\triangle \phi[n]=$ $\angle y[n]-\angle y[n-1]$, respectively, between two consecutively received symbols. However, in pursuit of an improved maximum achievable error-free transmission rate and/or an increased resilience against the formation of a high-Doppler-induced error-floor, one has to exploit the correlation between the amplitude and phase distortions experienced by the consecutively transmitted symbols with the aid of multiple-symbol-based detection, i.e. by using multiple-symbol differential detection (MSDD) [4]. As another benefit, it is worthwhile noting that the MSDD is also capable of increasing the iterative gain attained by the turbo receiver in the context of channel-coded systems. This is because the generation of soft-information by the MSDSD for the bits within the same detection window benefits from exploiting each other's improved-confidence reliability information provided by the channel decoder. As a result, the enhanced iterative gain attained by the MSDSD-aided turbo receiver may be translated to an increased error-free transmission rate for DAPSK-modulated systems, as it will be demonstrated in the following sections.

\section{A. MAP-Based Multiple-Symbol Differential Detection}

1) Principle of the MSDD: Basically, the MSDD makes a decision about the $k_{N}$ th block of $(N-1)$ consecutively transmitted DAPSK symbols $\mathbf{x}\left[k_{N}\right]=\left[x\left[k_{N}(N-1)\right], \cdots, x\left[\left(k_{N}+1\right)(N-1)\right]\right]^{\mathrm{T}}$ on the basis of $N$ successively received symbols stored in $\mathbf{y}\left[k_{N}\right]=$ $\left[y\left[k_{N}(N-1)\right], \cdots, y\left[\left(k_{N}+1\right)(N-1)\right]\right]^{\mathrm{T}}$. Since each element of $\mathbf{x}\left[k_{N}\right]$ is the product of the component DASK and DPSK symbols, we have $\mathbf{x}\left[k_{N}\right]=\mathbf{a}\left[k_{N}\right] \cdot \mathbf{s}\left[k_{N}\right]$ with the vectors $\mathbf{a}\left[k_{N}\right]$ and $\mathbf{s}\left[k_{N}\right]$ containing the corresponding $N$ consecutively transmitted constituent DASK and DPSK symbols, respectively. Thus, a multiple-symbolbased transmission may be ready to mode as follows:

$$
\begin{aligned}
\mathbf{y}\left[k_{N}\right] & =\mathbf{X}_{\mathrm{d}}\left[k_{N}\right] \mathbf{h}\left[k_{N}\right]+\mathbf{w}\left[k_{N}\right], \\
& =\mathbf{A}_{\mathrm{d}}\left[k_{N}\right] \mathbf{S}_{\mathrm{d}}\left[k_{N}\right] \mathbf{h}\left[k_{N}\right]+\mathbf{w}\left[k_{N}\right],
\end{aligned}
$$

where $\mathbf{X}_{\mathrm{d}}\left[k_{N}\right]=\operatorname{diag}\left\{\mathbf{x}\left[\mathrm{k}_{\mathrm{N}}\right]\right\}, \mathbf{A}_{\mathrm{d}}\left[k_{N}\right]=\operatorname{diag}\left\{\mathbf{a}\left[k_{N}\right]\right\}$ and $\mathbf{S}_{\mathrm{d}}\left[k_{N}\right]=\operatorname{diag}\left\{\mathbf{s}\left[k_{N}\right]\right\}$ are all diagonal matrices with their first upper-left element being the reference DAPSK symbol $x\left[k_{N}(N-\right.$ $1)] \triangleq x_{\text {ref }} \in \mathcal{X}$, the reference component DASK symbol $a\left[k_{N}(N-1)\right] \triangleq a_{\mathrm{ref}} \in \mathcal{A}$ and the reference component DPSK symbol $s\left[k_{N}(N-1)\right] \triangleq s_{\text {ref }} \in \mathcal{S}$, respectively. Additionally, $\mathbf{h}\left[k_{N}\right]=\left[h\left[k_{N}(N-1)\right], \cdots, h\left[\left(k_{N}+1\right)(N-1)\right]\right]^{\mathrm{T}}$ and $\mathbf{w}\left[k_{N}\right]=$ $\left[w\left[k_{N}(N-1)\right], \cdots, w\left[\left(k_{N}+1\right)(N-1)\right]\right]^{\mathrm{T}}$ of (3) represent the fading coefficients' column vector obeying a complex-valued Gaussian distribution $\mathcal{C N}\left(0, \Sigma_{h}\right)$ and the Gaussian noise column vector having a distribution of $\mathcal{C N}\left(0,2 \sigma_{w}^{2} \mathbf{I}_{N}\right)$, respectively. The channel's covariance matrix $\Sigma_{h}$ characterizes the fading correlation with the element located in the $n$th row and $m$ th column being $\varphi[m-n]$.

Under the assumption that both the fading and noise are zero-mean complex Gaussian processes, the probability density function (PDF) of $\mathbf{y}\left[k_{N}\right]$ conditioned both on $\boldsymbol{\Gamma}\left[k_{N}\right]=\left[\gamma\left[k_{N}(N-1)\right], \cdots, \gamma\left[\left(k_{N}+\right.\right.\right.$ 1) $(N-1)-1]]^{\mathrm{T}}$ and on $\boldsymbol{\Theta}\left[k_{N}\right]=\left[\theta\left[k_{N}(N-1)\right], \cdots, \theta\left[\left(k_{N}+\right.\right.\right.$ 1) $(N-1)-1]]^{\mathrm{T}}$, i.e. $p\left(\mathbf{y}\left[k_{N}\right] \mid \boldsymbol{\Gamma}\left[k_{N}\right], \boldsymbol{\Theta}\left[k_{N}\right]\right)$, can be expressed by averaging $p\left(\mathbf{y} \mid \mathbf{X}_{d}\right)$ over all possible values of $x_{\text {ref }}$ as follows (the block index $k_{N}$ is omitted for notation simplicity):

$$
\begin{aligned}
p(\mathbf{y} \mid \boldsymbol{\Gamma}, \boldsymbol{\Theta}) & =\mathcal{E}_{x_{\text {ref }}}\left\{p\left(\mathbf{y} \mid \mathbf{X}_{\mathrm{d}}\right)\right\} \\
& =\mathcal{E}_{x_{\text {ref }}}\left\{\frac{\exp \left\{-\mathbf{y}^{\mathrm{H}}\left[\Psi\left(\mathbf{X}_{\mathrm{d}}\right)\right]^{-1} \mathbf{y}\right\}}{\pi^{N} \operatorname{det}\left[\Psi\left(\mathbf{X}_{\mathrm{d}}\right)\right]}\right\},
\end{aligned}
$$

where the conditional autocorrelation matrix

$$
\Psi\left(\mathbf{X}_{\mathrm{d}}\right)=\mathcal{E}\left\{\mathbf{y y}^{\mathrm{H}} \mid \mathbf{X}_{\mathrm{d}}\right\}=\mathbf{X}_{\mathrm{d}} \Sigma_{h} \mathbf{X}_{\mathrm{d}}^{\mathrm{H}}+2 \sigma_{w}^{2} \mathbf{I}_{N}
$$

is dependent on the transmitted signal matrix $\mathbf{X}_{\mathrm{d}}$. With the aid of the Bayes' theorem, the soft bit information in terms of aposteriori LLRs can be calculated at the output of the MAP-MSDD as:

$$
\begin{aligned}
L_{D}\left(b_{i}^{n} \mid \mathbf{y}\right) & =\ln \frac{\operatorname{Pr}\left(b_{i}^{n}=+1 \mid \mathbf{y}\right)}{\operatorname{Pr}\left(b_{i}^{n}=-1 \mid \mathbf{y}\right)} \\
& =\ln \frac{\sum_{\mathbf{b} \in \mathbb{B}_{n, i,+1}} p(\mathbf{y} \mid \boldsymbol{\Gamma}, \boldsymbol{\Theta}) \operatorname{Pr}(\mathbf{b})}{\sum_{\mathbf{b} \in \mathbb{B}_{n, i,-1}} p(\mathbf{y} \mid \boldsymbol{\Gamma}, \boldsymbol{\Theta}) \operatorname{Pr}(\mathbf{b})},
\end{aligned}
$$

where $\mathbb{B}_{n, i, \pm 1}$ represents the set of $2^{(p N-1)}$ legitimate transmitted bit vectors $\mathbf{b}$ associated with the $i$ th bit of the $p$-bit-coded symbol being $b_{i}^{n}= \pm 1(i \in\{0, \cdots, p-1\})$. In the sequel, the extrinsic LLR can be obtained by excluding the corresponding a priori LLR, $L_{A}\left(b_{i}^{n}\right)=\ln \frac{\operatorname{Pr}\left(b_{i}^{n}=+1\right)}{\operatorname{Pr}\left(b_{i}^{n}=-1\right)}$, from the aposteriori LLR, $L_{D}\left(b_{i}^{n} \mid \mathbf{y}\right)$, which is exploited by the outer channel decoder after passing it through the deinterleaver as shown in Fig. 1.

2) Complexity of the MAP-MSDD: According to (5) and (9), the asymptotic complexity of the MAP-MSDD of a $2^{p}$-DAPSK scheme using $2^{q}$ concentric rings is $\mathcal{O}\left(p \cdot 2^{(p N)}\right)$. Therefore, employing the brute-force search carried out by the MAP-MSDD, might impose a potentially excessive computational complexity and hence may preclude its practical implementation, especially for high-order modulation schemes and/or for high observation window sizes. For example, under the assumption of an observation window size of $N=6$ and the 16-DAPSK scheme $(p=1, q=4)$, the number of evaluations of the PDF $p(\mathbf{y} \mid \boldsymbol{\Gamma}, \boldsymbol{\Theta})$ of (5) required for each 4-bit-coded symbol is as high as $2^{26}=6.7109 \times 10^{7}$. 


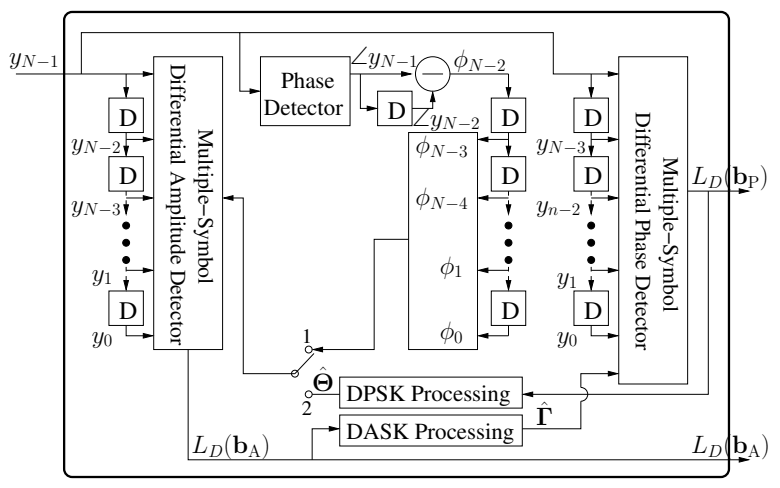

Fig. 2. Iterative Multiple-Symbol Differential Amplitude/Phase Detection (Illustration for the first multiple-symbol block, i.e., $k_{N}=0$ ).

\section{B. The Design of Iterative Amplitude/Phase MSDD}

Recently, the SD mechanism has been successfully adopted for the MAP-MSDD of a DPSK modulated system [7] based on the fact that the transmitted signal matrix $\mathbf{X}_{\mathrm{d}}$ is unitary. This technique achieved a significant complexity reduction. Unfortunately, this SDaided complexity reduction scheme cannot be directly applied for the DAPSK modulated system considered, since $\mathbf{X}_{\mathrm{d}}$ is no longer unitary. As another approach of reducing the complexity, the idea of decoupling the joint amplitude and phase detection was conceived in [9] for MSDD invoked for uncoded DAPSK for transmission over the AWGN channel. Regretfully, only a limited complexity reduction was achieved by this sub-optimum scheme at the cost of a compromised performance. From this section, we commence tackling the challenging issue of implementing MAP-MSDD for the DAPSK scheme at a substantially reduced complexity.

In order to recover this potentially substantial performance degradation imposed by the sub-optimum scheme proposed in [9], here a novel iterative A/P MSDD (IAP-MSDD) mechanism is proposed for channel coded DAPSK modulated systems, where specifically tailored information may be iteratively exchanged between the decoupled serially concatenated multiple-symbol differential amplitude detector (MSDAD) and multiple-symbol differential phase detector (MSDPD), as illustrated in Fig. 2. To be specific, $N$ consecutively received symbols are collected and fed through both the MSDAD and MSDPD of Fig. 2, where the soft-decision-based detection of the amplitude- and phase-modulation-related bits is carried out independently and iteratively. In the presence of the transmit-domain phase information $\hat{\boldsymbol{\Theta}}$, the aposteriori amplitude-modulation-related bit LLRs may be computed by the MSDAD detector as:

$$
\begin{aligned}
L_{D}\left(b_{\gamma, i}^{n} \mid \mathbf{y}, \hat{\boldsymbol{\Theta}}\right) & =\ln \frac{\operatorname{Pr}\left(b_{\gamma, i}^{n}=+1 \mid \mathbf{y}, \hat{\boldsymbol{\Theta}}\right)}{\operatorname{Pr}\left(b_{\gamma, i}^{n}=-1 \mid \mathbf{y}, \hat{\boldsymbol{\Theta}}\right)} \\
& =\ln \frac{\sum_{\mathbf{b}_{\gamma} \in \mathbb{B}_{n, i,+1}^{\gamma}} p(\mathbf{y} \mid \boldsymbol{\Gamma}, \hat{\boldsymbol{\Theta}}) \operatorname{Pr}(\boldsymbol{\Gamma} \mid \hat{\boldsymbol{\Theta}})}{\sum_{\mathbf{b}_{\gamma} \in \mathbb{B}_{n, i,-1}^{\gamma}} p(\mathbf{y} \mid \boldsymbol{\Gamma}, \hat{\boldsymbol{\Theta}}) \operatorname{Pr}(\boldsymbol{\Gamma} \mid \hat{\boldsymbol{\Theta}})}, \\
& =\ln \frac{\sum_{\mathbf{b}_{\gamma} \in \mathbb{B}_{n, i,+1}^{\gamma}} p(\mathbf{y} \mid \boldsymbol{\Gamma}, \hat{\boldsymbol{\Theta}}) \operatorname{Pr}\left(\mathbf{b}_{\gamma}\right)}{\sum_{\mathbf{b}_{\gamma} \in \mathbb{B}_{n, i,-1}^{\gamma}} p(\mathbf{y} \mid \boldsymbol{\Gamma}, \hat{\boldsymbol{\Theta}}) \operatorname{Pr}\left(\mathbf{b}_{\gamma}\right)},
\end{aligned}
$$

where $\mathbb{B}_{n, i, \pm 1}^{\gamma}$ represents the set of $2^{[q(N-1)-1]}$ legitimate amplitudemodulation-related MSB vectors $\mathbf{b}_{\gamma}$ associated with $b_{\gamma, i}^{n}= \pm 1$ ( $i \in\{1, \cdots, q\}$ ). Initially, when the phase information $\hat{\boldsymbol{\Theta}}$ is not available from the MSDPD, the initial phase information is obtained based on the output of the phase detector as $\hat{\boldsymbol{\Theta}}=\left[\phi_{0}, \cdots, \phi_{(N-1)}\right]^{T}$ by toggling the phase information feedback switch to the ' 1 ' location of Fig. 2, in order to neglect the phase error in the first round of MSDAD detection. Then, the amplitude ratios stored in $\hat{\boldsymbol{\Gamma}}$ may be calculated relying on the DASK processing of the aposteriori amplitude-modulation-related bit LLRs, i.e. $L_{D}\left(\mathbf{b}_{\gamma} \mid \mathbf{y}, \hat{\boldsymbol{\Theta}}\right)$ of (10), which are next delivered to the serially concantenated MSDPD. Similarly, with the aid of the amplitude ratio estimates $\hat{\boldsymbol{\Gamma}}$, the aposteriori phase-modulation-related bit LLRs $L_{D}\left(\mathbf{b}_{\theta} \mid \mathbf{y}, \hat{\boldsymbol{\Gamma}}\right)$ may be computed by the MSDPD as follows:

$$
L_{D}\left(b_{\theta, i}^{n} \mid \mathbf{y}, \hat{\boldsymbol{\Gamma}}\right)=\ln \frac{\sum_{\mathbf{b}_{\theta} \in \mathbb{B}_{n, i,+1}^{\theta}} p(\mathbf{y} \mid \hat{\boldsymbol{\Gamma}}, \boldsymbol{\Theta}) \operatorname{Pr}\left(\mathbf{b}_{\theta}\right)}{\sum_{\mathbf{b}_{\theta} \in \mathbb{B}_{n, i,-1}^{\theta}} p(\mathbf{y} \mid \hat{\boldsymbol{\Gamma}}, \boldsymbol{\Theta}) \operatorname{Pr}\left(\mathbf{b}_{\theta}\right)},
$$

where $\mathbb{B}_{n, i, \pm 1}^{\theta}$ denotes the set of $2^{[(p-q)(N-1)-1]}$ legitimate phasemodulation-related bit vectors $\mathbf{b}_{\theta}$ associated with $b_{\theta, i}^{n}= \pm 1(i \in$ $\{1, \cdots, p-q\}$ ). From the second iteration of the MSDAD process, the phase information feedback switch is toggled to the '2' position, since $\hat{\boldsymbol{\Theta}}$ in (10) can be computed based on the DPSK processing of the aposteriori phase-modulation-related bit LLRs, $L_{D}\left(\mathbf{b}_{\theta} \mid \mathbf{y}, \hat{\boldsymbol{\Gamma}}\right)$ of (13), delivered by the MSDPD, as observed in Fig. 2, in the interest of exploiting the improved-confidence phase information in the MSDAD detection. We note that both the amplitude ratio and phase difference estimates, i.e., $\hat{\boldsymbol{\Gamma}}$ and $\hat{\boldsymbol{\Theta}}$, can be obtained in either hard-decision or soft-decision manner from the corresponding aposteriori bit LLRs.

In our investigations we found that the conditional autocorrelation matrix $\Psi\left(\mathbf{X}_{\mathrm{d}}\right)$ is dependent on $a_{\text {ref }}$, but not on $s_{\text {ref }}$. Hence, a further complexity reduction may be achieved by averaging $p\left(\mathbf{y} \mid \mathbf{X}_{\mathrm{d}}\right)$ over all possible $a_{\text {ref }}$ values instead of $x_{\text {ref }}$, when computing the $p(\mathbf{y} \mid \boldsymbol{\Gamma}, \boldsymbol{\Theta})$ of (5). Thus, the burden of computing $p(\mathbf{y} \mid \boldsymbol{\Gamma}, \hat{\boldsymbol{\Theta}})$ in (12) and $p(\mathbf{y} \mid \hat{\boldsymbol{\Gamma}}, \boldsymbol{\Theta})$ in (13) can be reduced by a factor of $2^{(p-q)}$ using

$$
p(\mathbf{y} \mid \hat{\boldsymbol{\Gamma}}, \boldsymbol{\Theta})=\mathcal{E}_{a_{\text {ref }}}\left\{p\left(\mathbf{y} \mid \mathbf{X}_{\mathrm{d}}\right)\right\},
$$

instead of using (5). Consequently, the asymptotic complexity per iteration of the proposed IAP MSDD scheme for the $2^{p}$-DAPSK scheme using $2^{q}$ concentric-ring constellation is $\mathcal{O}\left(q \cdot 2^{(q N)}+(p-q)\right.$. $\left.\left.2^{(p-q)(N-1)+q}\right)\right)$. Hence, under the same assumption of $N=6$ and that of 16-DAPSK, the number of evaluations of (5) required for each symbol in the IAP-MSDD process becomes $2^{6}+3 \times 2^{16}=1.9667 \times$ $10^{5}$ per iteration $(99.97 \%$ of the total complexity is contributed by the phase-modulation-related bit detection). This total complexity is two orders of magnitude lower than that of the traditional full-searchbased MSDD.

\section{Complexity Reduction Strategies For the IAP-MSDD}

Although a substantial complexity reduction can be attained, the complexity imposed by the IAP-MSDD of Fig. 2 proposed for the DAPSK scheme may still be deemed to be excessive, as illustrated in Section III-B, thus preventing its implementation in most pratical scenarios. Hence, we continue our quest for more efficient complexity reduction techniques designed for the IAP-MSDD, in particular for its computationally more demanding MSDPD stage, which contributes the majority of the total complexity imposed.

\section{A. Estimation of the Transmit-Domain Symbol Amplitude}

According to (14), an immediate further complexity reduction by a factor of $2^{q}$ may be achieved at the MSDPD stage, if the amplitudes $\hat{\mathbf{A}}_{\mathrm{d}}$ of the transmitted symbols, instead of the amplitude ratios $\hat{\boldsymbol{\Gamma}}$, are estimated on the basis of the aposteriori LLRs $L_{D}\left(\mathbf{b}_{\gamma}^{n} \mid \mathbf{y}, \hat{\boldsymbol{\Theta}}\right)$ provided by the MSDAD detector. This is because in the presence of the amplitude estimates $\hat{\mathbf{A}}_{\mathrm{d}}$, the MSDPD detector may become capable of approximately computing the aposteriori LLRs $L_{D}\left(\mathbf{b}_{\theta}^{n} \mid \mathbf{y}, \hat{\boldsymbol{\Gamma}}\right)$ without averaging over all possible amplitudes of the reference symbol by using

$$
\begin{aligned}
L_{D}\left(b_{\theta, i}^{n} \mid \mathbf{y}, \hat{\boldsymbol{\Gamma}}\right) & \approx L_{D}\left(b_{\theta, i}^{n} \mid \mathbf{y}, \hat{\mathbf{A}}_{\mathrm{d}}\right), \\
& =\ln \frac{\sum_{\mathbf{b}_{\theta} \in \mathbb{B}_{n, i,+1}^{\theta}} p\left(\mathbf{y} \mid \hat{\mathbf{A}}_{\mathrm{d}}, \boldsymbol{\Theta}\right) \operatorname{Pr}\left(\mathbf{b}_{\theta}\right)}{\sum_{\mathbf{b}_{\theta} \in \mathbb{B}_{n, i,-1}^{\theta}} p\left(\mathbf{y} \mid \hat{\mathbf{A}}_{\mathrm{d}}, \boldsymbol{\Theta}\right) \operatorname{Pr}\left(\mathbf{b}_{\theta}\right)},
\end{aligned}
$$

where we have

$$
p\left(\mathbf{y} \mid \hat{\mathbf{A}}_{\mathrm{d}}, \boldsymbol{\Theta}\right)=p\left[\mathbf{y} \mid \tilde{\mathbf{X}}_{\mathrm{d}}=\hat{\mathbf{A}}_{\mathrm{d}} \times \mathbf{S}_{\mathrm{d}}(\boldsymbol{\Theta})\right],
$$


with the diagonal matrix $\mathbf{S}_{\mathrm{d}}(\boldsymbol{\Theta})$ containing the $N$-component DPSK symbols along its diagonal associated with the phase difference information $\Theta$. Bearing in mind the benefit of acquiring the transmitdomain symbol amplitude estimates, let us now further elaborate on the amplitude estimation procedure, which is constituted by the following two major steps:

Step 1: Estimate the amplitude, $a_{\text {ref }}$, of the reference symbol, namely, the first symbol $x_{0}$, of the block of $N$ successively transmitted symbols on the basis of the amplitude ratio $\hat{\Gamma}$ and phase difference estimates $\hat{\boldsymbol{\Theta}}$, provided by the MSDAD and MSDPD detectors, respectively.

By exploiting the fact that at the transmitter the equi-probable amplitudes of the reference symbol of a specific multiple-symbol block is independent of both the information-carrying amplitude ratios as well as of the phase differences among the successively transmitted symbols, we have $\operatorname{Pr}\left(a_{\text {ref }}=\alpha^{k} \mid \hat{\boldsymbol{\Gamma}}, \hat{\boldsymbol{\Theta}}\right)=\operatorname{Pr}\left(a_{\text {ref }}=\right.$ $\left.\alpha^{k}\right)=2^{-q}, k \in\left\{0, \cdots, 2^{q}-1\right\}$. Then, by exploiting Bayes' theorem, the soft-decision-based amplitude of the reference symbol may be calculated as follows:

$$
\begin{aligned}
\hat{a}_{\mathrm{ref}} & =\sum_{k=0}^{2^{q}-1} \alpha^{k} \cdot \operatorname{Pr}\left(\check{a}_{\mathrm{ref}}=\alpha^{k} \mid \mathbf{y}, \hat{\boldsymbol{\Gamma}}, \hat{\boldsymbol{\Theta}}\right) \\
& =\sum_{k=0}^{2^{q}-1} \alpha^{k} \cdot \frac{p\left(\mathbf{y} \mid \check{a}_{\mathrm{ref}}=\alpha^{k}, \hat{\boldsymbol{\Gamma}}, \hat{\boldsymbol{\Theta}}\right) \operatorname{Pr}\left(\check{a}_{\mathrm{ref}}=\alpha^{k} \mid \hat{\boldsymbol{\Gamma}}, \hat{\boldsymbol{\Theta}}\right)}{p(\mathbf{y} \mid \hat{\boldsymbol{\Gamma}}, \hat{\boldsymbol{\Theta}})} \\
& =\sum_{k=0}^{2^{q}-1} \frac{\alpha^{k} \cdot p\left(\mathbf{y} \mid \check{a}_{\mathrm{ref}}=\alpha^{k}, \hat{\boldsymbol{\Gamma}}, \hat{\boldsymbol{\Theta}}\right) \operatorname{Pr}\left(\check{a}_{\mathrm{ref}}=\alpha^{k}\right)}{\sum_{l=0}^{2^{q}-1} p\left(\mathbf{y} \mid \check{a}_{\mathrm{ref}}=\alpha^{l}, \hat{\boldsymbol{\Gamma}}, \hat{\boldsymbol{\Theta}}\right) \operatorname{Pr}\left(\check{a}_{\mathrm{ref}}=\alpha^{l}\right)}, \\
& =\sum_{k=0}^{2^{q}-1} \frac{\alpha^{k} \cdot p\left(\mathbf{y} \mid \check{a}_{\mathrm{ref}}=\alpha^{k}, \hat{\boldsymbol{\Gamma}}, \hat{\boldsymbol{\Theta}}\right)}{\sum_{l=0}^{2^{q}-1} p\left(\mathbf{y} \mid \check{a}_{\mathrm{ref}}=\alpha^{l}, \hat{\boldsymbol{\Gamma}}, \hat{\boldsymbol{\Theta}}\right)} \\
& =\sum_{k=0}^{2^{q}-1} \frac{\alpha^{k} \cdot p\left(\mathbf{y} \mid \hat{\mathbf{A}}_{\mathrm{d}}^{k}, \hat{\boldsymbol{\Theta}}\right)}{\sum_{l=0}^{2^{q}-1} p\left(\mathbf{y} \mid \hat{\mathbf{A}}_{\mathrm{d}}^{l}, \hat{\boldsymbol{\Theta}}\right)}
\end{aligned}
$$

where the diagonal matrix $\hat{\mathbf{A}}_{\mathrm{d}}^{k}$ contains the amplitude estimates of the transmitted symbols along its diagonal associated with its first diagonal element $a_{0}=\check{a}_{\text {ref }}=\alpha^{k}$. Then, the diagonal elements of $\hat{\mathbf{A}}_{\mathrm{d}}^{k}$ may be calculated recursively as:

$$
\hat{a}_{n+1}=\sum_{\check{\gamma}_{n+1}, \check{a}_{n} \in \mathcal{A}} \alpha^{\left(i_{\mathrm{A}}\left\{\check{\gamma}_{n+1}\right\}+i_{\mathrm{A}}\left\{\check{a}_{n}\right\}\right) \bmod 2^{q}} \operatorname{Pr}\left(\check{\gamma}_{n+1}\right) \operatorname{Pr}\left(\check{a}_{n}\right),
$$

where the ASK symbol probability $\operatorname{Pr}\left(\check{\gamma}_{n+1}\right)$ may be readily calculated based on the aposteriori ASK-modulation-related bit-LLRs, i.e., on $L_{D}\left(\mathbf{b}_{\gamma}^{n+1} \mid \mathbf{y}, \hat{\mathbf{\Theta}}\right)$ of (10) generated by the MSDAD, while the DASK symbol probability $\operatorname{Pr}\left(\breve{a}_{n}\right)$ can be approximately evaluated as:

$$
\operatorname{Pr}\left(\check{a}_{n}=\alpha^{k}\right) \approx \begin{cases}1, & \text { for } \hat{a}_{n} \leq \alpha^{k}, k=0 ; \\ \frac{\hat{a}_{n}-\alpha^{k-1}}{\alpha^{k+1}-\alpha^{k}}, & \text { for } \alpha^{k-1} \leq \hat{a}_{n} \leq \alpha^{k} ; \\ \frac{\alpha^{k+1}-\hat{a}_{n}}{\alpha^{k+1}-\alpha^{k}}, & \text { for } \alpha^{k}<\hat{a}_{n}<\alpha^{k+1} \\ 0, & \text { for all the other cases, }\end{cases}
$$

which essentially reduces the computational complexity imposed by (19), especially when the size of $\mathcal{A}$ is high.

Step 2: Upon obtaining the amplitude estimate for the reference symbol from (18), we estimate the amplitudes of the remaining $(N-$ 1) transmitted symbols of the specific multiple-symbol block with the aid of the amplitude ratio estimates $\hat{\Gamma}$.

More specifically, in order to generate $\hat{\mathbf{A}}_{\mathrm{d}}$ for the MSDPD detection of (15), the soft-decision-based amplitude calculation criterion of (19) - which was employed when obtaining $\hat{\mathbf{A}}_{\mathrm{d}}^{k}$ of (18) - is also invoked for recursively computing the diagonal elements of the matrix $\hat{\mathbf{A}}_{\mathrm{d}}$ commencing from the first element $\hat{a}_{\text {ref }}$ of (18).

\section{B. Incorporating a Structured Tree Search in the MSDPD Stage}

As another benefit of estimating the amplitudes of the transmitted symbols, an efficiently structured tree search employed by the wellknown SD may be incorporated into the computationally demanding MSDPD stage, as detailed in this section. We will demonstrate that this technique is capable of achieving a further significant complexity reduction. Provided that the amplitude estimate matrix $\hat{\mathbf{A}}_{\mathrm{d}}$ has been obtained, we now further elaborate on (17) by reformulating it as follows (the argument $\boldsymbol{\Theta}$ in $\mathbf{S}_{\mathrm{d}}(\boldsymbol{\Theta})$ is omitted for notational simplicity):

$$
p\left(\mathbf{y} \mid \tilde{\mathbf{X}}_{d}=\hat{\mathbf{A}}_{\mathrm{d}} \mathbf{S}_{\mathrm{d}}\right)=\frac{\exp \left\{-\mathbf{y}^{\mathrm{H}}\left[\Psi\left(\tilde{\mathbf{X}}_{\mathrm{d}}\right)\right]^{-1} \mathbf{y}\right\}}{\pi^{N} \operatorname{det}\left[\Psi\left(\tilde{\mathbf{X}}_{\mathrm{d}}\right)\right]},
$$

where according to (7) we have:

$$
\begin{aligned}
\Psi\left(\tilde{\mathbf{X}}_{\mathrm{d}}\right) & =\hat{\mathbf{A}}_{\mathrm{d}} \mathbf{S}_{\mathrm{d}} \Sigma_{h}\left(\hat{\mathbf{A}}_{\mathrm{d}} \mathbf{S}_{\mathrm{d}}\right)^{\mathrm{H}}+2 \sigma_{w}^{2} \mathbf{I}_{N}, \\
& =\mathbf{S}_{\mathrm{d}}\left(\tilde{\Sigma}_{h}+2 \sigma_{w}^{2} \mathbf{I}_{N}\right) \mathbf{S}_{\mathrm{d}}^{\mathrm{H}},
\end{aligned}
$$

with $\tilde{\Sigma}_{h} \triangleq \hat{\mathbf{A}}_{\mathrm{d}} \Sigma_{h} \hat{\mathbf{A}}_{\mathrm{d}}^{\mathrm{H}}$ being termed as the equivalent channel covariance matrix. Note that for a given $\hat{\mathbf{A}}_{\mathrm{d}}$, the denominator of (21) is independent of $\mathbf{S}_{\mathrm{d}}$, since $\mathbf{S}_{\mathrm{d}}$ is unitary, i.e. we have $\mathbf{S}_{\mathrm{d}}^{-1}=\mathbf{S}_{\mathrm{d}}^{\mathrm{H}}$. Thus, with the aid of the Max-log approximation, the calculation of the aposteriori phase-modulation-related bit LLRs of (16) may be simplified as:

$$
\begin{gathered}
L_{D}\left(\hat{b}_{\theta, i}^{n}\right) \approx \max _{\mathbf{b}_{\theta} \in \mathbb{B}_{n, i,+1}^{\theta}}\left\{-\mathbf{y}^{\mathrm{H}}\left[\Psi\left(\tilde{\mathbf{X}}_{\mathrm{d}}\right)\right]^{-1} \mathbf{y}+\ln \operatorname{Pr}(\boldsymbol{\Theta})\right\} \\
-\max _{\mathbf{b}_{\theta} \in \mathbb{B}_{n, i,-1}^{\theta}}\left\{-\mathbf{y}^{\mathrm{H}}\left[\Psi\left(\tilde{\mathbf{X}}_{\mathrm{d}}\right)\right]^{-1} \mathbf{y}+\ln \operatorname{Pr}(\boldsymbol{\Theta})\right\} .
\end{gathered}
$$

Furthermore, since $\mathbf{S}_{\mathrm{d}}$ is unitary and owing to the independence of the elements of $\Theta,(24)$ can be reformulated as follows:

$$
\begin{gathered}
L_{D}\left(\hat{b}_{\theta, i}^{n}\right) \approx \max _{\mathbf{b}_{\theta} \in \mathbb{B}_{n, i,+1}^{\theta}}\left\{-\left\|\mathbf{L}^{\mathrm{T}} \mathbf{Y}_{\mathrm{d}}^{\mathrm{H}} \mathbf{s}\right\|^{2}+\sum_{n=0}^{N-2} \ln \operatorname{Pr}\left(\theta_{n}\right)\right\} \\
-\max _{\mathbf{b}_{\theta} \in \mathbb{B}_{n, i,-1}^{\theta}}\left\{-\left\|\mathbf{L}^{\mathrm{T}} \mathbf{Y}_{\mathrm{d}}^{\mathrm{H}} \mathbf{s}\right\|^{2}+\sum_{n=0}^{N-2} \ln \operatorname{Pr}\left(\theta_{n}\right)\right\},
\end{gathered}
$$

where we have $\mathbf{Y}_{\mathrm{d}} \triangleq \operatorname{diag}\{\mathbf{y}\}$ and the lower triangular matrix $\mathbf{L}$ satisfying $\mathbf{L} \mathbf{L}^{\mathrm{H}}=\left(\tilde{\Sigma}_{h}+2 \sigma_{w}^{2} \mathbf{I}_{N}\right)^{-1}$ can be obtained by the Cholesky factorization of the symmetric positive definite matrix $\left(\tilde{\Sigma}_{h}+2 \sigma_{w}^{2} \mathbf{I}_{N}\right)^{-1}$ of (23). By defining the upper triangular matrix $\mathbf{U}=\mathbf{L}^{\mathrm{T}} \mathbf{Y}_{\mathrm{d}}^{\mathrm{H}}$ and after a few straightforward manipulations, we finally arrive at:

$$
\begin{aligned}
& L_{D}\left(\hat{b}_{\theta, i}^{n}\right) \approx \max _{\mathbf{b}_{\theta} \in \mathbb{B}_{n, i,+1}^{\theta}}\left\{\sum_{n=0}^{N-2}\left(\ln \operatorname{Pr}\left(\theta_{n}\right)-\sum_{m=n}^{N-1}\left|u_{n m} s_{m}\right|^{2}\right)\right\} \\
& -\max _{\mathbf{b}_{\theta} \in \mathbb{B}_{n, i,-1}^{\theta}}\left\{\sum_{n=0}^{N-2}\left(\ln \operatorname{Pr}\left(\theta_{n}\right)-\sum_{m=n}^{N-1}\left|u_{n m} s_{m}\right|^{2}\right)\right\}
\end{aligned}
$$

where $u_{n m}$ is the element of matrix $\mathbf{U}$ located in the $n$th row and $m$ th column. Consequently, thanks to the upper-triangular structure of the matrix $\mathbf{U}$, we may find the two maximum values in (26) with the aid of the efficient tree-search-based SD algorithm of [7].

\section{Performance Evaluation and Discussions}

In order to visualize the EXtrinsic Information Transfer (EXIT) charateristics of the proposed IAP-MSDSD scheme, in Fig. 3 we plot the EXIT curves associated with different observation window sizes of $N$ for the IAP-MSDSD against those of the CDD and of the traditional MSDD. Under the assumption of the 16-DAPSK modulated system of Fig. 1 and a normalized Doppler frequency of $f_{d}=0.01$, the resultant EXIT curves seen in Fig. 3 are obtained by evaluating the extrinsic mutual information (MI), $I_{E}$, at the output of the specific differential detector for a given input stream of bit 


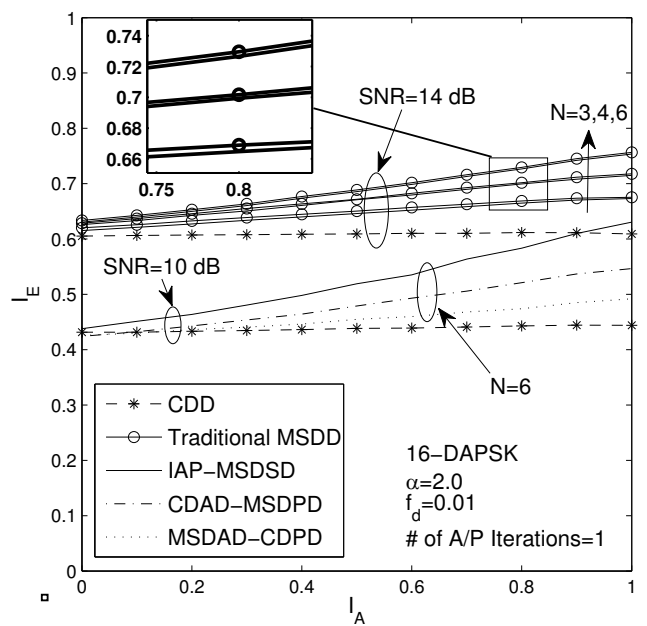

Fig. 3. EXIT chart of the IAP-MSDSD employed in the 16-DAPSK system.

LLRs along with the a priori MI $I_{A}$ at SNRs of 10 and $14 \mathrm{~dB}$. According to the area properties of the EXIT chart, the upwardsshifted EXIT curve of the IAP-MSDSD in Fig. 3 suggests that a significantly higher maximum transmission rate may be achieved in comparison to the CDD assisted system using $N_{\text {wind }}=2$. The throughput gain achieved by jointly detecting $N>2$ data symbols using the IAP-MSDSD is also visualized in the 3D plot of Fig. 4, where the maximum achievable throughput of the IAP-MSDSD-aided 16-DAPSK modulated system is depicted versus both the SNR and the ring-ratio $\alpha$. Additionally, the dotted and dot-dashed EXIT curves of Fig. 3 suggest that a compromise may be struck between the maximum achievable rate and the complexity imposed with the aid of a hybrid detection mechanism, namely by the combined conventional differential amplitude detection (CDAD) and MSDPD as well as by the amalgamated MSDAD and conventional differential phase detection (CDPD). Moreover, as implied by the small gap between the EXIT curve of the IAP-MSDSD and that of the traditional MSDD seen in Fig. 3, both the MSDAD and MSDPD of the IAPMSDSD of Fig. 2 has to be invoked only once, in order to approach the performance of the traditional MSDD. Hence, this observation allows us to set the number of iterations between the MSDAD and MSDPD stages to one in our simulations throughout the paper in order to avoid any unnecessary operations. Thus, remarkablely, the complexity imposed by the IAP-MSDSD becomes about five orders of magnitude lower than that of the traditional MSDSD in the context of the 16-DAPSK modulation-aided system across a wide range of SNRs, as seen in Fig. 5, where the complexity quantified in terms of the number of transmitted symbol vector candidate enumerations during the differential detection is portrayed versus both the SNR and the ring-ratio $\alpha$. Furthermore, observe from the IAP-MSDSDrelated throughput and complexity surfaces plotted in Figs. 4 and 5, respectively, that the ring-ratio $\alpha$ employed by 16-DAPSK plays a crucial role in determining both the system's achievable transmission rate as well as its detection complexity. Specifically, the simulation results seen in Figs. 4 and 5 suggest that setting the ring-ratio to $\alpha \approx 2.0$ constitutes an appropriate choice for maximizing the achievable throughput, while minimizing the complexity imposed by the proposed IAP-MSDSD scheme.

In conclusion, we proposed an IAP-MSDSD scheme for DAPSK modulated systems in this paper, which was shown to be capable of achieving a near-MAP-MSDD performance at a substantially reduced complexity, that was about five orders of magnitude lower than that imposed by the traditional MAP-MSDD in the case of 16-DAPSK.

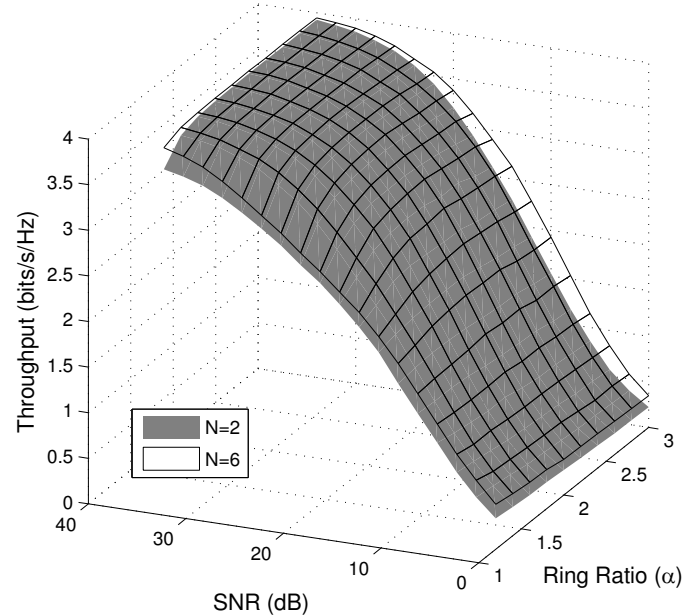

Fig. 4. Maximum arhievahle throuchnut of the 16-nADCK evetem

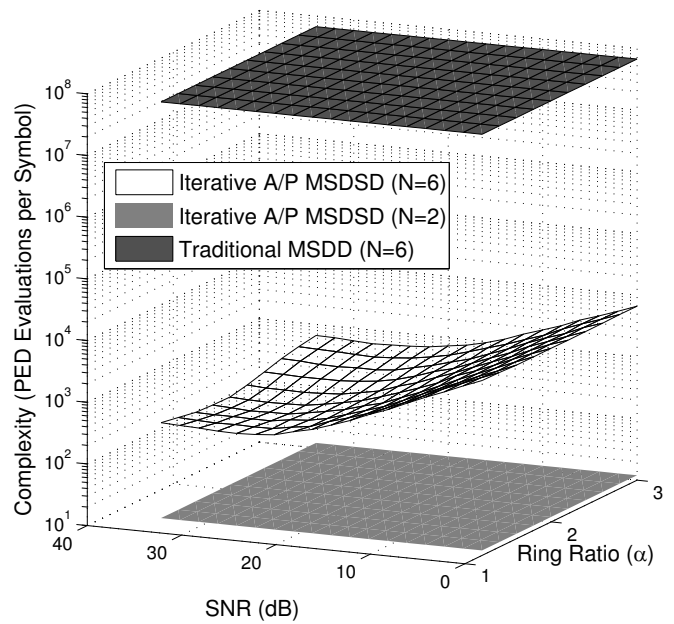

Fig. 5. Complexity reduction achieved by the IAP-MSDSD for 16-DAPSK.

\section{REFERENCES}

[1] W. T. Webb, L. Hanzo, and R. Steele, "Bandwidth efficient QAM schemes for Rayleigh fading channels," IEE Proceedings I, Communications, Speech and Vision, pp. 169-175, June 1991.

[2] H. Rohling and V. Engels, "Differential amplitude phase shift keying (DAPSK) - a new modulation method for DTVB," Proceedings of International Broadcasting Convention, pp. 102-108, 1995.

[3] L. Hanzo, S. X. Ng, W. Webb, and T. Keller, Quadrature Amplitude Modulation: From Basics to Adaptive Trellis-Coded, Turbo-Equalised and Space-Time Coded OFDM, CDMA and MC-CDMA Systems. WileyBlackwell, 2004.

[4] M. Machida, S. Handa, and S. Oshita, "Multiple-symbol differential detection of APSK based on MAP criterion," IEEE Global Telecommunications Conference, pp. 2740-2744, Nov. 1998.

[5] K. Ishibashi, H. Ochiai, and R. Kohno, "Low-complexity bit-interleaved coded DAPSK for Rayleigh-fading channels," IEEE Journal on Selected Areas in Communications, pp. 1728-1738, Sept. 2005.

[6] R. F. H. Fischer, L. H. J. Lampe, and S. H. M. Weinfurtner, "Coded modulation for noncoherent reception with application to OFDM," IEEE Transactions on Vechicular Technology, pp. 910-919, July 2001.

[7] V. Pauli, L. Lampe, and R. Schober, "“Turbo DPSK” using soft multiplesymbol differential sphere decoding," IEEE Transactions on Information Theory, vol. 52, no. 4, pp. 1385-1398, 2006.

[8] L. Hanzo, Y. Akhtman, L. Wang, and M. Jiang, MIMO-OFDM for LTE, WIFI and WIMAX: Coherent versus Non-Coherent and Cooperative Turbo-Transceivers. John Wiley and IEEE Press, 2010.

[9] T. Suzuki and T. Mizuno, "Multiple-symbol differential detection scheme for differential amplitude modulation," Mobile Communications Advanced Systems and Components, Lecture Notes in Computer Science, pp. 196207, 1994. 